\title{
Collaboration of FlhF and FlhG to regulate polar- flagella number and localization in Vibrio alginolyticus
}

\author{
Correspondence \\ Michio Homma \\ g44416a@cc.nagoya-u.ac.jp
}

Received 23 August 2007

Revised 24 January 2008

Accepted 26 January 2008

\author{
Akiko Kusumoto, ${ }^{1}$ Akari Shinohara, ${ }^{1,2}$ Hiroyuki Terashima, ${ }^{1}$ Seiji Kojima, ${ }^{1,2}$ \\ Toshiharu Yakushi, ${ }^{1,2} \dagger$ and Michio Homma ${ }^{1,2}$
${ }^{1}$ Division of Biological Science, Graduate School of Science, Nagoya University, Chikusa-Ku, Nagoya 464-8602, Japan \\ ${ }^{2}$ Soft Nano-Machine Project, CREST, JST, Japan
}

\section{INTRODUCTION}

Many motile bacteria have flagella that rotate by means of a motor embedded in the cytoplasmic membrane and create a driving force by rotating a helical filament, like a screw, connected to the motor. The number and localization of flagella are different among species. Escherichia coli, Salmonella typhimurium (Macnab, 1996) and Bacillus subtilis (Kearns \& Losick, 2003) have peritrichous flagella. Vibrio cholerae (Prouty et al., 2001), Caulobacter crescentus (Brun et al., 1994) and Pseudomonas aeruginosa (Tsuda \& Iino, 1983) have a single polar flagellum. Vibrio fischeri (Millikan \& Ruby, 2004), Helicobacter pylori (Niehus et al., 2004) and Pseudomonas putida (Harwood et al., 1989) have multiple flagella at the pole. Vibrio alginolyticus and Vibrio parahaemolyticus have both a single polar flagellum and peritrichous flagella (McCarter, 2001). The flagella are not essential, and cells must expend significant energy for their maintenance. Bacteria minimize the cost of producing flagella, and it seems that one strategy involves the precise regulation of flagellar number.

The flagellar genes are conserved among peritrichously flagellated and polarly flagellated bacteria, and gene regulation and morphogenesis are also similar among

tPresent address: Department of Bioscience and Biotechnology, Faculty of Agriculture, Shinshu University, 8304 Minamiminowa, Nagano 399-4598, Japan. various species (Macnab, 1996; Wu \& Newton, 1997; Prouty et al., 2001; McCarter, 2001). The flagella, which are composed of a filament, hook and basal body, are formed from the proximal end towards the distal tip. During flagellar morphogenesis, first the MS ring of the basal body, which is composed of FliF, is established on the cytoplasmic membrane (Kubori et al., 1992). Next, the switch complex or the $\mathrm{C}$ ring, which is composed of three proteins, FliG, FliM and FliN, is assembled under the MS ring. Then, the specific apparatus for protein export is assembled inside the $\mathrm{C}$ ring to form the entrance of the channel for flagellar proteins (Kubori et al., 1997; Macnab, 2004). The rod proteins are exported by way of this apparatus and the rod structure is constructed. Then, the other proteins, such as the hook protein and flagellin are exported similarly and polymerized into tubular structures that extend from the rod. Formation of the MS ring is thought to initiate the flagellar assembly, so the number and location of MS-ring complexes are likely to determine the number and location of flagella.

The flagellar genes are hierarchically expressed under strict control. In Vibrio and Pseudomonas, they are classified into at least three classes: early genes (master regulator); intermediate genes, the expression of which depends on $\sigma^{54}$ (MS ring, hook, basal body, switch, export apparatus, transcriptional regulators including FlaL, FlaM, FlhF and FlhG, che proteins, $\sigma^{28}$ and flagellin); and late genes, the 
expression of which depends on $\sigma^{28}$ (flagellins, motor proteins and FlgM) (Dasgupta et al., 2003; McCarter, 2001; Prouty et al., 2001). The intermediate class is further divided into two classes: the FlaK-dependent genes (homologues are FleQ in P. aeruginosa and FlrA in $V$. cholerae) that encode the MS ring, switch, export apparatus, FlaL, FlaM, FlhF, FlhG and $\sigma^{28}$; and the FlaLM-dependent genes (homologues are FleSR in $P$. aeruginosa and FlrBC in $V$. cholerae) that encode hook protein, rod proteins, LP-ring proteins and flagellin. These four gene classes are called class I (early genes), class II (FlaK-dependent intermediate genes), class III (FlaLMdependent intermediate genes) and class IV (late genes).

In $V$. cholerae, $P$. aeruginosa and $P$. putida, overexpression of FlhF results in an increased number of polar flagella, and an $f l h F$ gene disruption gives a reduced number and aberrant placement of flagella in $V$. cholerae, $P$. aeruginosa and P. putida (Correa et al., 2005; Murray \& Kazmierczak, 2006; Pandza et al., 2000). Moreover, FlhF has been reported to increase the expression of class III genes in $V$. cholerae (Correa et al., 2005). Therefore, it has been suggested that FlhF increases the number of flagella by promoting the expression of flagellar genes. However, it is unclear how FlhF determines the location of the flagella. In $V$. cholerae and $P$. aeruginosa, overexpression of FlhG results in a reduced number of flagella and gives a nonflagellated phenotype, and an $f h G$ gene disruption gives a hyperflagellated phenotype (Correa et al., 2005; Dasgupta et al., 2000). In Pseudomonas, it has been shown that the FleN protein (the homologue is FlhG in Pseudomonas sp.) binds to the flagellar-gene-specific transcriptional regulator, FleQ (Dasgupta \& Ramphal, 2001), which regulates the transcription of class II genes with $\sigma^{54}$ (Dasgupta et al., 2002). The FleN protein represses transcription of class II genes and of the fleN gene by itself via FleQ. The FleQ homologue in $V$. cholerae, FlrA, also regulates transcription of class II genes (Prouty et al., 2001).

We have previously reported a multi-polar flagellar mutant of $V$. alginolyticus, KK148, and we identified the mutation responsible, which is a nonsense mutation in the flh $G$ gene (Kusumoto et al., 2006). We cloned the upstream gene, $f l h F$, and the $f l h G$ gene. These genes are unique to polarflagellated bacteria, although FlhF and FlhG have similarity with the E. coli signal recognition particle (SRP) receptor FtsY and the E. coli cell division inhibitor MinD, respectively (Kusumoto et al., 2006). Overexpression of FlhF results in an increased number of polar flagella; on the other hand, overexpression of FlhG results in a reduced number of polar flagella and gives a non-flagellated phenotype. These results are consistent with those of earlier studies with $V$. cholerae and Pseudomonas (Correa et al., 2005; Dasgupta et al., 2000; Murray \& Kazmierczak, 2006; Pandza et al., 2000). Moreover, co-expression of FlhF and FlhG reduces the number of polar flagella more significantly than expression of FlhG alone, implying that FlhG works together with FlhF to regulate the number of polar flagella (Kusumoto et al., 2006).
In this study, we show that the polar localization of FlhF is reduced by FlhG, independent of other flagellar proteins, and that FlhF and FlhG interact with each other. We speculate that the FlhF-FlhG interaction inhibits FlhF from localizing at the pole and that flagellation is thus suppressed. These results contribute to our understanding of the control of flagellar number and the location of the flagellum in mono-polar flagellar systems.

\section{METHODS}

Bacterial strains and growth conditions. $V$. alginolyticus and $E$. coli strains used in this study are listed in Table 1. V. alginolyticus cells were cultured at $30{ }^{\circ} \mathrm{C}$ in VC medium $[0.5 \%(\mathrm{w} / \mathrm{v})$ Tryptone, $0.5 \%$ $(\mathrm{w} / \mathrm{v})$ yeast extract, $0.4 \%(\mathrm{w} / \mathrm{v}) \mathrm{K}_{2} \mathrm{HPO}_{4}, 3 \%(\mathrm{w} / \mathrm{v}) \mathrm{NaCl}, 0.2 \%(\mathrm{w} / \mathrm{v})$ glucose] or in VPG medium [1\%(w/v) Tryptone, $0.4 \%(\mathrm{w} / \mathrm{v})$ $\mathrm{K}_{2} \mathrm{HPO}_{4}, 3 \%(\mathrm{w} / \mathrm{v}) \mathrm{NaCl}, 0.5 \%(\mathrm{w} / \mathrm{v})$ glycerol]. E. coli cells were cultured at $37{ }^{\circ} \mathrm{C}$ in LB medium $[1 \%(\mathrm{w} / \mathrm{v})$ Tryptone, $0.5 \%(\mathrm{w} / \mathrm{v})$ yeast extract, $0.5 \%(\mathrm{w} / \mathrm{v}) \mathrm{NaCl}$. When necessary, the following antibiotics were used: chloramphenicol $\left(2.5 \mu \mathrm{g} \mathrm{ml} \mathrm{m}^{-1}\right.$ for $V$. alginolyticus or $25 \mu \mathrm{g} \mathrm{ml}^{-1}$ for E. coli), kanamycin $\left(100 \mu \mathrm{g} \mathrm{ml}^{-1}\right)$ and ampicillin $\left(50\right.$ or $100 \mu \mathrm{g} \mathrm{ml}^{-1}$ ).

DNA manipulations and sequencing. Routine DNA manipulations were carried out according to standard procedures. Restriction endonucleases and other enzymes for DNA manipulations were purchased from TaKaRa Shuzo, Toyobo, and New England Biolabs. Nucleotide sequences were determined using the BigDye Terminator v3.1 Cycle Sequencing kit (Applied Biosystems) and an ABI PRISM 3100-Avant Genetic Analyzer (Applied Biosystems).

Gene disruption. The $f l h F$ and $f l h F G$ deletion strains LPN1 and LPN2 were generated from VIO5 cells, which are defective for production of lateral flagella and wild-type with respect to the polar flagellum, by homologous recombination, as described previously (Terashima et al., 2006). The suicide vectors containing the sacB gene, pKY704- $\Delta$ flhF-sacB and pKY704- $\Delta$ flhFG-sacB, were used for this construction. First, for homologous recombination of the $f l h F$ and flhFG genes, $1073 \mathrm{bp}$ downstream of the $f l h G$ gene, containing the fliA gene and $313 \mathrm{bp}$ from the beginning of the cheY gene, were cloned into a cloning vector, $\operatorname{pGEM} 5 \mathrm{Zf}(+)$, and sequenced. Using the resultant plasmids and primers based on these sequences, we made inframe deletions in $f l h F$ or $f l h G$ (DNA encoding 475 aa was deleted from $f l h F$ and DNA encoding 772 aa was deleted from flhFG), which were integrated into the chromosome of VIO5 cells by homologous recombination. Next, strains that had undergone the first recombination were cultured in VC medium without antibiotics overnight, and then screened for the second recombination based on their sucrose sensitivity. Finally, the $f l h F$ and $f l h F G$ deletions in the chromosome were confirmed by PCR. The flhF deletion and flhFG double deletion strains were named LPN1 and LPN2, respectively.

Transformation of Vibrio cells. $V$. alginolyticus cells were transformed by electroporation as described previously (Kawagishi et al., 1994). The cells were subjected to osmotic shock and washed thoroughly with $20 \mathrm{mM} \mathrm{MgSO}$. Electroporation was carried out according to the manufacturer's instructions using a Gene Pulser electroporation apparatus (Japan Bio-Rad Laboratories) at an electric field strength of $5.0-7.5 \mathrm{kV} \mathrm{cm}^{-1}$.

High-intensity dark-field microscopy. Flagella were observed using a dark-field microscope (Olympus model BHT) equipped with a $100 \mathrm{~W}$ mercury lamp (Ushio USH-102). An image was recorded 
Table 1. Bacterial strains and plasmids used in this study

Abbreviations: $\mathrm{Amp}^{\mathrm{r}}$, ampicillin resistant; $\mathrm{Cm}^{\mathrm{r}}$, chloramphenicol resistant; Rif ${ }^{\mathrm{r}}$, rifampicin resistant; Pof ${ }^{+}$, possessing polar flagellum; Laf ${ }^{-}$, lacking lateral flagella; Multi-Pof, possessing multiple polar flagella.

\begin{tabular}{|c|c|c|}
\hline Strain or plasmid & Genotype or description & Reference or source \\
\hline \multicolumn{3}{|l|}{ V. alginolyticus strains } \\
\hline VIO5 & VIK4 $\left(\right.$ Rif $^{\mathrm{r}}$ Pof $^{+}$Laf $)$ & Okunishi et al. (1996) \\
\hline KK148 & VIO5 $f l h G$ ( Rif $^{\mathrm{r}}$ Pof $^{+}$Laf Multi-Pof) & Kusumoto et al. (2006) \\
\hline YM14 & YM4 rpoN (Pof Laf $\left.^{-}\right)$ & Kawagishi et al. (1997) \\
\hline LPN1 & VIO5 $\Delta f l h F\left(\right.$ Rif $^{\mathrm{r}}$ Pof $^{+}$Laf $\left.^{-}\right)$ & This study \\
\hline LPN2 & VIO5 $\Delta f l h F G\left(\right.$ Rif $^{\mathrm{r}}$ Pof $^{+}$Laf $)$ & This study \\
\hline \multicolumn{3}{|l|}{ E. coli strains } \\
\hline $\mathrm{DH} 5 \alpha$ & & Grant et al. (1990) \\
\hline BL21(DE3)pLysS & T7 expression host & Studier (1991) \\
\hline \multicolumn{3}{|l|}{ Plasmids } \\
\hline pHFS401 & $s a c B$ in $\mathrm{pSU} 41$ & Terashima et al. (2006) \\
\hline pKY704 & $\mathrm{Cm}^{\mathrm{r}}$, suicide vector & Xu et al. (1994) \\
\hline pKY704- $\Delta f l h F-s a c B$ & $1425 \mathrm{bp}$-deleted $f l h F$ fragment in pKY704 & This study \\
\hline $\mathrm{pKY} 704-\Delta f l h F G-s a c B$ & 2316 bp-deleted $f l h F G$ fragment in pKY704 & This study \\
\hline pET-3d & $A m p^{r}, T 7$ expression vector & Novagen \\
\hline $\mathrm{pET}-f l h F$ & his $_{6}-$ tev-flhF in pET-3d & This study \\
\hline pGEX-6P-2 & $\mathrm{Amp}^{\mathrm{r}}, \mathrm{P}_{\mathrm{tac}}, g s t$ & GE Healthcare \\
\hline pGEX-flhG13 & $g s t-f l h G$ in pGEX-6P-2 & This study \\
\hline pBAD24 & $A m p^{r}, P_{B A D}$ & Guzman et al. (1995) \\
\hline pBAD33 & $\mathrm{Cm}^{\mathrm{r}}, \mathrm{P}_{\mathrm{BAD}}$ & Guzman et al. (1995) \\
\hline pAK322 & $f l h F$ in $\mathrm{pBAD} 33$ & Kusumoto et al. (2006) \\
\hline pAK520 & $f l h G$ in $\mathrm{pBAD} 33$ & Kusumoto et al. (2006) \\
\hline pAK721 & $f l h F G$ in $\mathrm{pBAD} 33$ & Kusumoto et al. (2006) \\
\hline pAK325 & flhF-egfp in pBAD33 & This study \\
\hline pAK541 & flhG-egfp in pBAD33 & This study \\
\hline
\end{tabular}

using a charge-coupled device (CCD) camera (Sony model SSCM370) and a DVD video recorder (Panasonic model DMR-E100H).

Electron microscopy. Samples were negatively stained with $2 \%$ $(\mathrm{w} / \mathrm{v})$ potassium phosphotungstate $(\mathrm{pH} 7.4)$, and then observed with a JEM-1200 EXII electron microscope (JEOL).

Fluorescence microscopy. Vibrio cells bearing a plasmid, pAK325 or pAK541, were cultured overnight in VC medium. The overnight culture was diluted $1: 100$ in VPG medium containing $0.02 \%(\mathrm{w} / \mathrm{v})$ arabinose and $2.5 \mu \mathrm{g}$ chloramphenicol ml $\mathrm{ml}^{-1}$, and incubated at $30{ }^{\circ} \mathrm{C}$ for $4 \mathrm{~h}$. Fluorescence microscopy was carried out with a 'tunnel slide', which is a rudimentary flow chamber constructed from a coverslip, a microscope slide and double-sided tape. Poly-L-lysine $(0.1 \%$, w/v) was loaded into a tunnel slide, and after $5 \mathrm{~min}$ the tunnel slide was washed with VPG medium. Cultures of the cells were applied by exchanging the medium, and then the tunnel slide was turned upsidedown and incubated for $1 \mathrm{~min}$ to adhere cells to the coverslip. The tunnel slides were washed with TMK buffer $(50 \mathrm{mM}$ Tris/ $\mathrm{HCl}$, $500 \mathrm{mM} \mathrm{KCl}, 5 \mathrm{mM}$ glucose, $5 \mathrm{mM} \mathrm{MgCl}_{2}, \mathrm{pH}$ 7.5) and observed under a BX-50 microscope (Olympus).

To stain polar flagella, cells were treated with the following preparation before observation. VPG medium containing antiserum raised against the polar flagellum (Fukuoka et al., 2005) was applied to the tunnel slide by exchanging the medium. After $3 \mathrm{~min}$ incubation, the tunnel slide was washed with VPG, and then medium containing a rhodamine-conjugated anti-rabbit-IgG antibody was added. After $3 \mathrm{~min}$ incubation, the tunnel slide was washed with VPG, then observed under a microscope. The images were recorded and processed using a digital camera (Hamamatsu Photonics, C474280-12AG) and imaging software (BD Bioscience, IPLab, version 3.9.5 r2 and Adobe Photoshop version 7)

Antibody raised against FlhF and FlhG. FlhF was purified from BL21 (DE3)/pLysS cells harbouring a plasmid producing hexahistidine $\left(\mathrm{His}_{6}\right)$-tagged FlhF $\left(\mathrm{His}_{6}-\mathrm{FlhF}\right)$, pET-flhF. Cells were harvested by centrifugation, washed with buffer A $[10 \mathrm{mM}$ Tris/ $\mathrm{HCl}, 150 \mathrm{mM}$ $\mathrm{NaCl}, 0.5 \mathrm{mM}$ PMSF, complete EDTA-free protease inhibitor (Roche), $\mathrm{pH} 8.0$ ], and then frozen at $-80{ }^{\circ} \mathrm{C}$. After thawing, the cells were resuspended in buffer A containing $10 \mu \mathrm{g}$ DNase I ml ${ }^{-1}$ and $5 \mathrm{mM} \mathrm{MgCl}_{2}$. The suspension was then passed twice through a French pressure cell at $500 \mathrm{~kg} \mathrm{~cm}^{-2}$. After centrifugation $(10000 \mathrm{~g}$ for $10 \mathrm{~min}), \mathrm{His}_{6}-\mathrm{FlhF}$ was present in the pellet as inclusion bodies. The pellet was resuspended in buffer A containing $4 \%(\mathrm{w} / \mathrm{v})$ Triton X100 , and then sonicated. The Triton-soluble membrane fraction was removed by centrifugation ( $10000 \mathrm{~g}$ for $10 \mathrm{~min}$ ). To remove the membrane fraction completely, the Triton-insoluble pellet was suspended again with buffer A containing Triton X-100, sonicated, and then centrifuged ( $10000 \mathrm{~g}$ for $10 \mathrm{~min}$ ). After the Triton-insoluble pellet had been rinsed twice with distilled water, the pellet was suspended in buffer A containing $8 \mathrm{M}$ urea, and incubated at $30{ }^{\circ} \mathrm{C}$ for $2 \mathrm{~h}$ with shaking. The suspension was centrifuged (7000 $\mathrm{g}$ for $15 \mathrm{~min}$ ), and then $\mathrm{His}_{6}-\mathrm{FlhF}$ was purified from the supernatant with $\mathrm{Ni}-\mathrm{NTA}$ resin.

FlhG was purified from $\mathrm{DH} 5 \alpha$ cells harbouring a plasmid producing glutathione $S$-transferase (GST)-fused FlhG, pGEX-flhG13. Cells were harvested by centrifugation, resuspended in buffer C (50 mM Tris/ $\mathrm{HCl}, 150 \mathrm{mM} \mathrm{NaCl}, 1 \mathrm{mM}$ EDTA, pH 8.0) containing $0.6 \mathrm{mg}$ 
lysozyme $\mathrm{ml}^{-1}$, and then incubated on ice for $30 \mathrm{~min}$. The cell suspension was passed twice through a French press at $500 \mathrm{~kg} \mathrm{~cm}^{-2}$. After removal of undisrupted cells by centrifugation $(10000 \mathrm{~g}$ for $10 \mathrm{~min}$ ), the insoluble fraction was removed by ultracentrifugation (100 $000 \mathrm{~g}$ for $1 \mathrm{~h}$ ). GST-fused FlhG in the soluble fraction was applied to a GSTrap FF column (GE Healthcare). After removal of the GST tag by applying PreScission protease (GE Healthcare), FlhG was eluted with buffer D (50 mM Tris, $150 \mathrm{mM} \mathrm{NaCl}, 1 \mathrm{mM}$ EDTA, $1 \mathrm{mM}$ DTT, pH 8.0).

Purified $\mathrm{His}_{6}-\mathrm{FlhF}$ and FlhG were separated by SDS-PAGE and stained with Coomassie blue R250. The band corresponding to each protein was excised and used to inoculate a rabbit. Rabbit anti-FlhG antibody was produced by Biogate.

Western blot analysis of flagellar proteins. V. alginolyticus cells were cultured overnight in VC medium. The overnight culture was diluted 1:100 in VPG medium, and then incubated at $30{ }^{\circ} \mathrm{C}$ for $4 \mathrm{~h}$. Cells were harvested by centrifugation, and then resuspended in double-distilled water. The cell suspensions were mixed with a onefifth volume of SDS loading buffer [0.2 M Tris/ $\mathrm{HCl}(\mathrm{pH} 6.8), 37.5 \%$ $(\mathrm{w} / \mathrm{v})$ glycerol, $6 \%(\mathrm{w} / \mathrm{v})$ SDS, $0.004 \%(\mathrm{w} / \mathrm{v})$ bromophenol blue] and a one-twentieth volume of 2-mercaptoethanol, and then boiled for $5 \mathrm{~min}$. Proteins in the samples were separated by SDS-PAGE and then electrophoretically transferred to a PVDF membrane (Millipore) using a semi-dry blotting apparatus (Bio-craft) according to the manufacturer's instructions. Immunoblotting was performed with anti-FlhF, anti-FlhG, anti-FlgI, anti-MotX, anti-MotY, anti-PomA, anti-PomB and anti-flagellin antibodies, as described previously (Nambu \& Kutsukake, 2000; Yagasaki et al., 2006; Fukuoka et al., 2005; Nishioka et al., 1998).

Immunoprecipitation. An overnight culture of $\triangle f l h F G$ cells harbouring a plasmid, pAK520 ( $f l h G)$ or pAK721 (flhFG), was diluted $1: 100$ into VPG medium containing $0.2 \%(\mathrm{w} / \mathrm{v})$ (for pAK520) or $0.01 \%(\mathrm{w} / \mathrm{v})$ (for pAK721) arabinose and $2.5 \mu \mathrm{g}$ chloramphenicol ml ${ }^{-1}$. After $4 \mathrm{~h}$ incubation at $30{ }^{\circ} \mathrm{C}$, the cells were harvested by centrifugation (10000 $g$ for $5 \mathrm{~min}$ ), suspended in buffer $\mathrm{E}[50 \mathrm{mM}$ Tris/HCl, $150 \mathrm{mM} \mathrm{NaCl}$, complete protease inhibitor (Roche), $\mathrm{pH}$ 7.5] to an $\mathrm{OD}_{660}$ of 10 , and sonicated to disrupt the cells. Undisrupted cells were removed by centrifugation (10000 $g$ for $5 \mathrm{~min}$ ). The supernatant was ultracentrifuged (100000 $\mathrm{g}$ for $1 \mathrm{~h}$ ). The supernatant from this step was diluted 1:5 into buffer E containing $0.1 \%(\mathrm{w} / \mathrm{v})$ Triton X-100, and anti-FlhF antibody and protein ASepharose CL-4B (GE Healthcare) were added. After $3 \mathrm{~h}$ incubation at $4{ }^{\circ} \mathrm{C}$, the protein A-Sepharose was washed four times with buffer $\mathrm{E}$ containing $0.1 \%(\mathrm{w} / \mathrm{v})$ Triton X-100, and was resuspended in buffer E containing 3\% (w/v) SDS, and boiled. The protein A-Sepharosebound materials were separated by SDS-PAGE, and FlhF and FlhG were detected by Western blot analysis. To eliminate the signal from heavy and light chains of the anti-FlhF antibody used for immunoprecipitation, the detection of FlhF was carried out with an ExtraCruz F kit (Santa Cruz).

\section{RESULTS}

\section{Phenotypes of the flhF deletion and the flhFG double deletion strains}

To investigate the function of FlhF, flhF and flhFG deletion strains were generated by means of homologous recombination. Electron microscopic observation revealed that the parent cells (VIO5) had a single polar flagellum (Fig. 1a), but we could not find any flagella on the $\Delta f l h F$ cells (Fig. 1c). This is consistent with the previous study, which

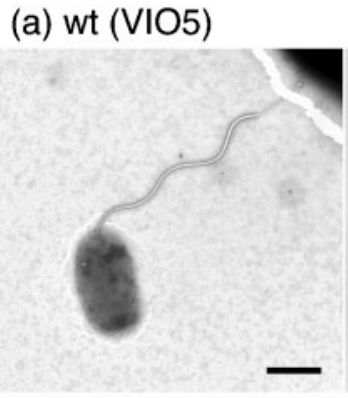

(c) $\Delta$ flhF (LPN1)

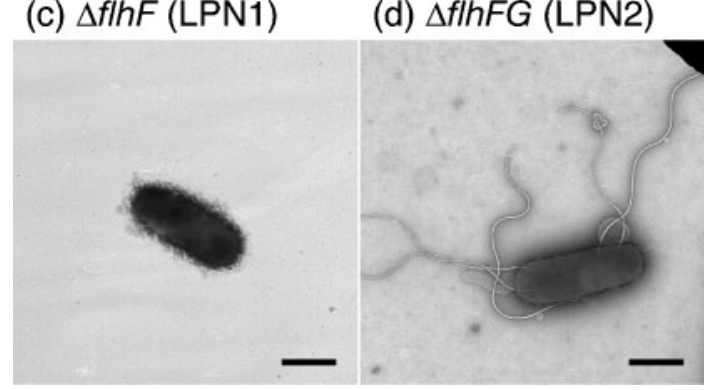

(b) flhG- (KK148)

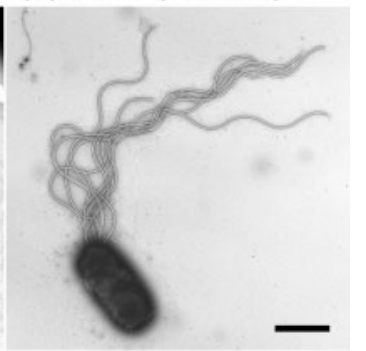

(d) $\triangle$ flhFG (LPN2)
Fig. 1. Electron micrographs of (a) VIO5 (wild-type; wt), (b) KK148 (flhG), (c) LPN1 ( $\Delta f / h F)$, and (d) LPN2 ( $\Delta f / h F G$ ) cells. Cells were negatively stained with potassium phosphotungstate. Bars, $1 \mu \mathrm{m}$.

showed that the overproduction of FlhF increases the number of polar flagella (Kusumoto et al., 2006). The $\Delta f l h F$ strain showed almost no swarming ability on $0.25 \%$ agar VPG plates after $6 \mathrm{~h}$ incubation (Fig. 2a, left). After $10 \mathrm{~h}$ incubation, however, swarming colonies of $\triangle f l h F$ cells showed definite expansion, although colonies of YM14 cells (an rpoN mutant that does not produce polar flagella) did not expand at all (Fig. 2a, right). This indicates that a very low percentage of the population of the $\Delta f l h F$ strain have flagella.

High-intensity dark-field microscopic observation of $\Delta$ flhFG cells indicated that most $(\sim 97.5 \%)$ were nonflagellated, although some of them $(\sim 2.5 \%)$ had several flagella. Electron microscopic observation of the $\triangle f l h F G$ cells also indicated that most were non-flagellated, although some had several flagella at lateral positions (Fig. 1d). Since the KK148 cells, which have a nonsense mutation in the flhG gene (Kusumoto et al., 2006), had multiple flagella at the pole (Fig. 1b), FlhF may be involved in regulating the flagellar number as well as the placement of the polar flagellum.

\section{Functions of the flhF and flhG genes}

The motility of $\triangle f l h F$ (LPN1) and $\triangle f l h F G$ (LPN2) cells expressing the $f l h F$ and/or $f h G$ genes on $0.25 \%$ agar VPG plates was tested (Fig. 2b). The $\Delta f l h F$ cells recovered their motility after expression of flhF in trans (Fig. 2b), and these cells also regained a polar flagellum, which was confirmed by high-intensity dark-field microscopy. $\triangle f l h F G$ cells 
(a)

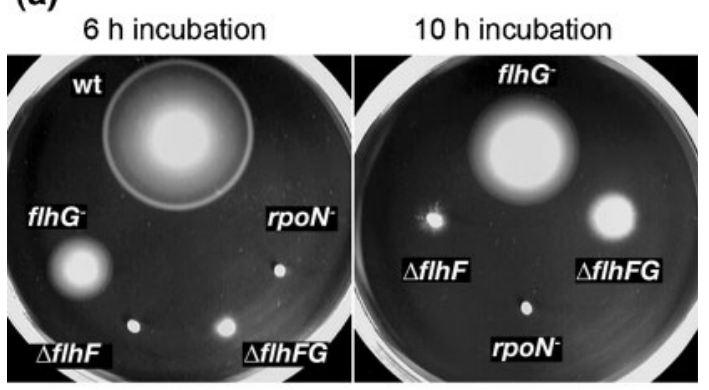

(b)

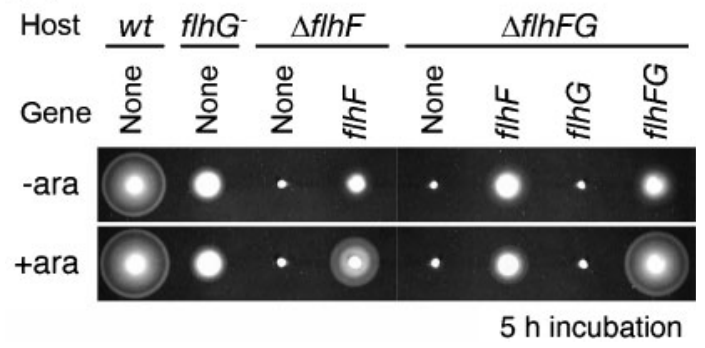

Fig. 2. Swarming ability of the $\Delta f / h F$ and $\Delta f / h F G$ cells. Swarming abilities are shown of the wild-type (wt; VIO5), $\Delta$ flhF (LPN1), $\Delta$ flhFG (LPN2), flhG mutant (KK148) and rpoN mutant (YM14) cells (a) without plasmids and (b) with plasmids pAK322 (flhF), pAK520 (flhG), pAK721 (flhFG) and pBAD33 (vector control). Aliquots $(0.5 \mu \mathrm{l})$ of overnight cultures were spotted onto $0.25 \%$ agar VPG plates without arabinose (a, left and right, or b, upper) or with $0.02 \%$ arabinose (b, lower), followed by incubation at $30{ }^{\circ} \mathrm{C}$ for $6 \mathrm{~h}$ (a, left), $10 \mathrm{~h}$ (a, right) or $5 \mathrm{~h}(\mathrm{~b})$.

expressing $f l h F$ recovered their motility even in the absence of arabinose (Fig. 2b), and the cells had multiple flagella at the cell pole, similar to the flhG mutant (KK148) cells. When the $f l h G$ gene was expressed in $\triangle f l h F G$ cells (Fig. 2b), no flagella were produced and motility was reduced to the level observed in $\Delta f l h F$ cells (data not shown). The expression of both $f l h F$ and $f l h G$ genes restored the motility of $\triangle f l h F G$ cells to some extent in the absence of inducer, and to the same level as that of the wild-type in the presence of $0.2 \%$ arabinose (Fig. $2 \mathrm{~b}$ ). Most of the cells had one or several polar flagella at the pole in the presence of $0.2 \%$ arabinose (data not shown).

\section{The amount of flagellar proteins in flhF and flhG mutants}

We evaluated the amount of various flagellar proteins in the wild-type cells, and in the $\Delta f l h F, f l h G, \Delta f l h F G$ and $r p o N$ mutant cells by means of Western blot analysis (Fig. 3). FlhF was not detected in the $\Delta f l h F, \Delta f l h F G$ or $r p o N$ cells (Fig. 3a), and FlhG was not detected in the flh $G, \Delta f l h F G$ or rpoN cells (Fig. 3b). These results confirmed that the flh $F$ gene, and the $f l h F$ and $f l h G$ genes in $\triangle f l h F$ and $\Delta f l h F G$ cells, respectively, were disrupted, and that the expression of

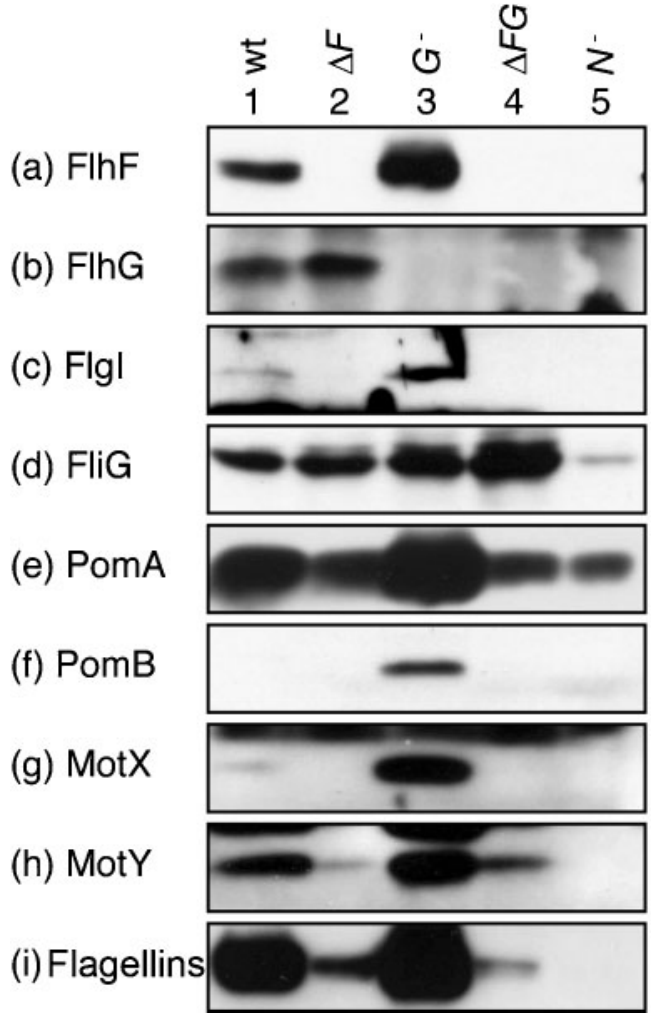

Fig. 3. Flagellar proteins in various mutant strains. Cells cultured overnight were recultured in VPG medium at $30{ }^{\circ} \mathrm{C}$ for $4 \mathrm{~h}$. Cells were harvested by centrifugation and resuspended in water. Proteins were separated by SDS-PAGE and immunoblotted using anti-FlhF (a), anti-FlhG (b), anti-Salmonella-Flgl (c), anti-FliG (d), anti-PomA (e), anti-PomB (f), anti-MotX (g), anti-MotY (h) and antiflagellin antibodies (i). Lanes: 1, VIO5 cells (wild-type; wt); 2, LPN1 cells $(\Delta f / h F ; \Delta F) ; 3$, KK148 cells (flhG; $\left.G^{-}\right)$; 4, LPN2 cells $(\Delta$ flhFG; $\Delta F G) ; 5$, YM14 cells $\left(r p o N ; N^{-}\right)$.

flhFG genes required $\sigma^{54}$, the $r p o N$ product. While a similar amount of FlhG was detected both in the wild-type and the $\Delta$ flhF cells (Fig. 3b), a larger amount of FlhF was detected in the flh $G$ mutant (Fig. 3a). In the flh $G$ mutant cells, most of the flagellar structural proteins (FlgI, PomA, PomB, MotX, MotY and flagellins), except for FliG, were detected in larger amounts than in the wild-type cells (Fig. 3c, e, f, g, $h, i)$, which is consistent with the number of flagella on the cells. A similar amount of FliG (intermediate class) was detected in the wild-type, $\Delta f h F$ and $f h G$ mutant cells, a larger amount was detected in the $\triangle f l h F G$ mutant, and a smaller amount was detected in the rpoN mutant (Fig. 3d). These results imply that the fliEFGHIJ operon, which is in the intermediate class, is most likely regulated by an additional mechanism besides $\sigma^{54}$ (RpoN).

\section{Subcellular localization of FlhF and FlhG}

To investigate the localization of FlhF and FlhG, GFP was fused to the $\mathrm{C}$ terminus of each protein. FlhF-GFP and FlhG-GFP were expressed in the $\Delta f l h F$ and $f l h G$ mutant 
cells, respectively, and polar flagella were visualized by means of immunofluorescence staining with an antibody raised against the polar flagellum (Fig. 4). The flagellated poles of cells showed the accumulation of FlhF-GFP as dots (Fig. 4a); on the other hand, flh $G$ mutant cells producing FlhG-GFP failed to generate flagella (Fig. $4 \mathrm{~b}$ ), suggesting that FlhF-GFP and FlhG-GFP have the same ability to increase or decrease the number of flagella as the intact FlhF and FlhG. FlhF-GFP was diffused throughout

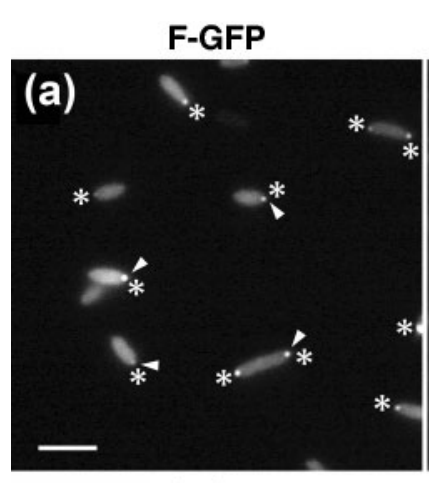

G-GFP
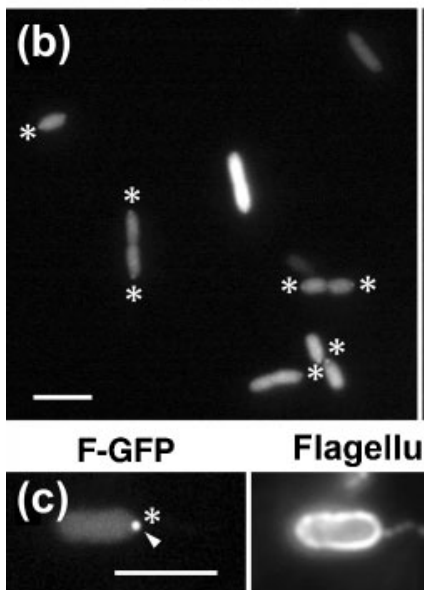

Flagellum
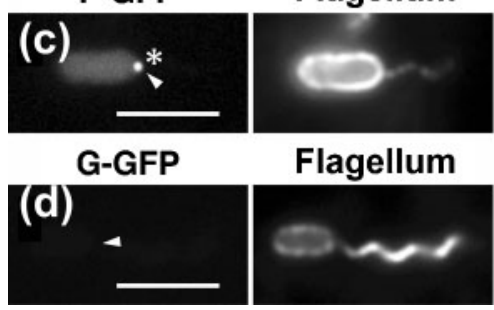

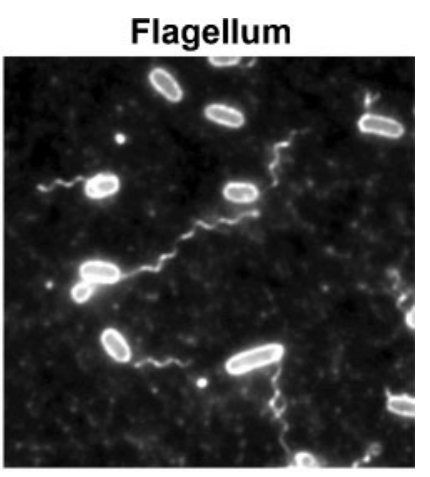

Flagellum

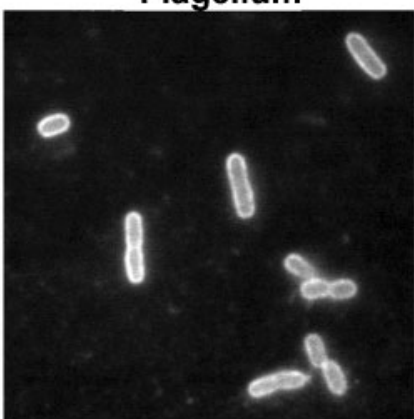

G-GFP Flagellum
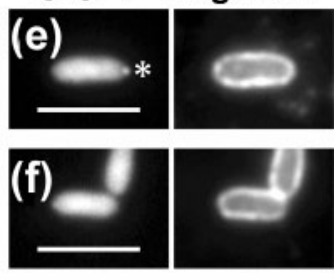

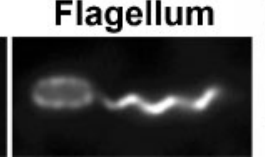

Fig. 4. Localization of GFP-fused FlhF (F-GFP) and FlhG (GGFP). $\Delta f / h F$ (LPN1) cells containing plasmid pAK325 (flhF-egfp) $(\mathrm{a}, \mathrm{c})$, and flhG mutant (KK148) cells containing plasmid pAK541 (flhG-egfp) (b, d, e, f), were observed by fluorescence microscopy. Cells cultured overnight were diluted 1:100 with VPG medium containing $0.02 \%(\mathrm{w} / \mathrm{v})$ arabinose. After $4 \mathrm{~h}$ incubation at $30{ }^{\circ} \mathrm{C}$, the cells were attached to a poly-L-lysine-coated cover glass. To identify the flagellated pole, the cells were labelled with anti-polarflagellum antiserum as the first antibody and rhodamine-conjugated anti-rabbit lgG antibody as the second antibody, and then observed under a fluorescence microscope. Asterisks indicate polar-localized F-GFP or G-GFP. Arrowheads indicate flagellated poles. Bars, $5 \mu \mathrm{m}$.

the cytoplasm, with some localization at one or both poles in the $\Delta f l h F$ cells (Fig. $4 \mathrm{a}, \mathrm{c}$ ). Most of the flagellated poles had FlhF-GFP foci (Fig. 4a, c). FlhG was also diffused throughout the cytoplasm in the flh $G$ mutant cells (Fig. 4b). FlhG-GFP was localized at the pole in $~ 30 \%$ of the cells (Fig. 4b, e, f). Most of the cells transformed with the flhG-gfp plasmid were not flagellated, and FlhGGFP was not detected when the cells were flagellated (Fig. 4d).

\section{Polar localization of FIhF is affected by FIhG}

The localization of FlhF-GFP and FlhG-GFP induced by arabinose was investigated in the $\triangle f l h F G$ and $r p o N$ mutant cells (Fig. 5). The amount of FlhF-GFP and FlhG-GFP in the cells did not differ among the strains, as confirmed by Western blotting (data not shown). FlhF-GFP was intensely localized at the pole in $\Delta$ flhFG cells (Fig. 5b), while FlhF-GFP was detected throughout the cytoplasm in addition to the poles in $\Delta f l h F$ cells (Fig. 5a). We analysed the ratio of fluorescent signal in the cytoplasm to that at the pole in FlhF-GFP-expressing cells (Fig. 5h). The signals at the pole (denoted as 'P') and in the cytoplasm (denoted as ' $\mathrm{C}$ ') were defined as the highest signal at the pole and the mean signal in the central cytoplasmic region of the cell, respectively (Fig. 5g). Fig. 5(h) shows the distribution of the ratio of $\mathrm{P}$ to $\mathrm{C}(\mathrm{P} / \mathrm{C})$. The $\mathrm{P} / \mathrm{C}$ of $\Delta f l h F$ cells ranged from 1.8 to 15.3 , and the mean \pm SD P/C was $5.1 \pm 3.2$. The $\mathrm{P} / \mathrm{C}$ of $\triangle$ flhFG cells ranged from 6.2 to 29.3 and the mean \pm SD P/C was $14.7 \pm 5.8$. These results clearly show that FlhF-GFP is strongly localized at the pole in $\triangle f l h F G$ cells compared with $\Delta f h F$ cells. Interestingly, the localization of FlhF in rpoN mutant cells, which do not express most of the flagellar proteins, including FlhF and FlhG, was similar to that in $\Delta f l h F$ cells (Fig. $5 c$ ). The P/C of $r p o N$ mutant cells ranged from 1.5 to 9.1 and the mean \pm SD P/C was $3.9 \pm 1.8$. This may suggest that other factor(s) whose expression depends on $\sigma^{54}$ are involved in the polar localization of FlhF. The FlhG-GFP signal was diffuse throughout the cytoplasm, although some FlhG-GFP formed fluorescent foci in flhG, $\Delta$ flhFG and $r p o N$ mutant cells (Fig. 5d, e, f). FlhG-GFP localized at $\sim 74 \%$ of the flh $G$ mutant cell poles, $\sim 82 \%$ of the $\triangle f l h F G$ cell poles, and $\sim 72 \%$ of the rpoN mutant cell poles. The localization of FlhG-GFP in $\triangle$ flhFG cells was similar to that in the flh $G$ mutant cells as well as that in $f l h F$ mutant cells. These results suggest that the polar localization of FlhF is inhibited by FlhG, while the localization of FlhG is not influenced by FlhF (see the model in Fig. 7).

To test whether the polar localization of FlhF is affected by the presence of FlhG in the absence of other Vibrio flagellar-related factors, GFP-fused FlhF or FlhG was produced with FlhG or FlhF in E. coli $\triangle$ flhDCBA cells, which lack the master regulators of the flagellar genes and do not express any flagellar genes, and in flagellated E. coli cells (RP437), which are wild-type for flagella formation 
(a) F-GFP $/ \triangle F$

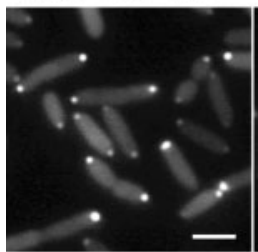

(b) F-GFP / $\triangle F G$

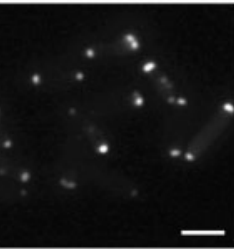

(d) G-GFP/G

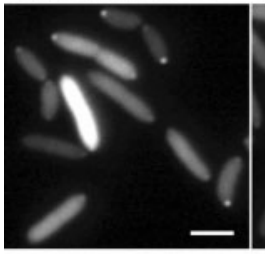

(e) G-GFP / $\triangle F G$

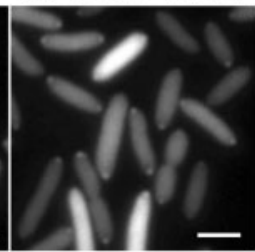

(g)

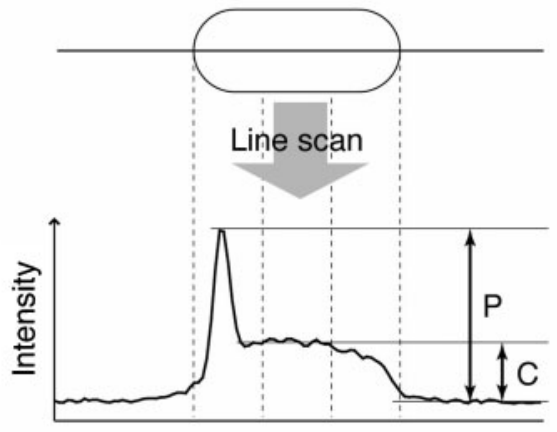

(h)

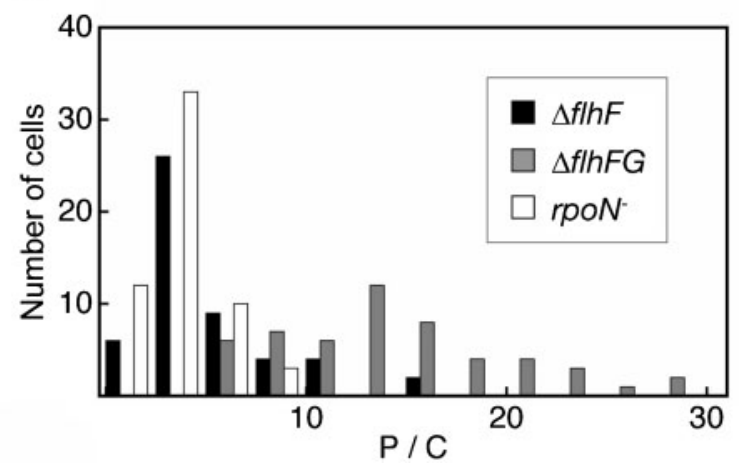

(data not shown). FlhF-GFP expression was diffuse throughout the cytoplasm and a fluorescent dot was detected at the pole of $\sim 36 \%$ of cells. When co-expressed with FlhG, the fluorescent dot was detected in only $\sim 2 \%$ of cells and the fluorescence was diffuse throughout the cytoplasm, showing that FlhF-GFP failed to localize at the pole. Polar localization of FlhF and reduction of FlhF polar localization was also observed in RP437 cells. These results support the idea that FlhF can localize to the pole in the absence of other flagellar-related factors and that FlhF interacts with FlhG. FlhG-GFP was diffuse throughout the cytoplasm and was not localized at the pole in E. coli $\triangle$ flhDCBA cells or RP437 cells. Therefore, the polar localization of FlhG seems to require specific factors present in Vibrio cells.
Fig. 5. The effect of FlhG on the polar localization of FlhF. The localization of FlhF-GFP (F-GFP) (a, b, c) and FlhG-GFP (G-GFP) $(\mathrm{d}, \mathrm{e}, \mathrm{f})$ in cells was observed. The host strains were as follows: (a) $\Delta f / h F\left(\Delta F\right.$, LPN1); (b, e) $\Delta f / h F G\left(\Delta F G\right.$, LPN2); (d) flhG mutant $\left(G^{-}\right.$, KK148); (c, f) rpoN mutant ( $N^{-}$, YM14). Cells cultured overnight were diluted 1:100 with VPG medium containing $0.02 \%$ arabinose. After $4 \mathrm{~h}$ incubation at $30{ }^{\circ} \mathrm{C}$, the cells were bound to a poly-L-lysine-coated cover glass. Cells were observed under a fluorescence microscope. Bars, $2 \mu \mathrm{m}$. Quantitative analysis of the effect of FlhG on polar localization of FlhF was performed $(\mathrm{g}, \mathrm{h})$. ( $\mathrm{g}$ ) Schematic illustration of the quantification procedure. Cells were scanned for fluorescence intensity along the major axis using the line scan mode of ImageJ analysis software (ImageJ version 1.37). The fluorescence intensity at the pole ('P') for one axis was obtained by subtracting the background value from the highest intensity (in arbitrary units) within the cell. The fluorescence intensity in the cytoplasm (' $\mathrm{C}$ ') for one axis was obtained in the same way, subtracting the background value from the average intensity of the cytoplasmic middle region (in arbitrary units) within the cell. (h) Histogram of the ratio of $\mathrm{C}$ to $\mathrm{P}(\mathrm{P} / \mathrm{C})$ of $51 \Delta \mathrm{flhF}$ (LPN1) (black bars), $53 \Delta f / h F G$ (LPN2) (grey bars), and $58 \mathrm{rpoN}$ mutant (YM14) (white bars) cells expressing FlhF-GFP.

\section{The FIhF-FIhG interaction}

Based on the observation that the polar localization of FlhF was inhibited by FlhG, we speculated that there is an interaction between FlhF and FlhG. Therefore, we attempted to directly demonstrate an FlhF-FlhG interaction. The $\triangle$ flhFG cells expressing flhG or flhFG genes were fractionated into soluble (cytoplasmic) and insoluble (membrane) fractions. FlhF was detected equally in the cytoplasmic fraction (Fig. 6a, lane 5) and in the insoluble fraction (Fig. 6a, lane 6) from $\triangle$ flhFG cells expressing both FlhF and FlhG. FlhG was also detected both in the cytoplasmic fraction (Fig. 6a, lanes 2 and 5) and the insoluble fraction (Fig. 6a, lanes 3 and 6), with more in the cytoplasmic fraction. There was no apparent difference in the localization profiles of FlhG when either flh $G$ or flhFG was co-expressed in $\triangle f l h F G$ cells.

As the cytoplasmic fraction was confirmed to contain both FlhF and FlhG, immunoprecipitation assays using an antiFlhF antibody were performed with the cytoplasmic fraction (Fig. 6b, lane 2). As a negative control, an immunoprecipitation assay was performed with the fraction containing only FlhG (Fig. 6b, lane 1). A significant amount of FlhG was immunoprecipitated with an anti-FlhF antibody from the cytoplasmic fraction containing FlhF and FlhG (Fig. 6b, lane 2), but not from that containing FlhG alone (Fig. 6b, lane 1), indicating an interaction between FlhF and FlhG.

\section{DISCUSSION}

From previous studies in V. cholerae and Pseudomonas, it has been suggested that FlhF and FlhG regulate the number of flagella by upregulating or downregulating the expression of 


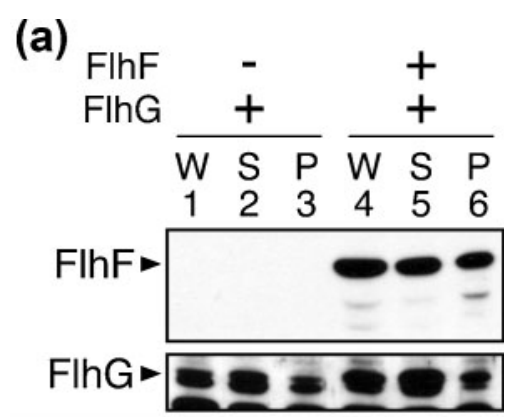

(b)

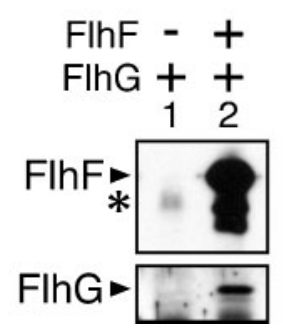

Fig. 6. Immunoprecipitation assay of the FlhF-FlhG complex using the anti-FlhF antibody. (a) $\Delta f / h F G$ (LPN2) cells expressing FlhG alone or both FlhF and FlhG were sonicated and then ultracentrifuged. FlhF and FlhG in the whole-cell lysate (W), the cytoplasmic fraction (supernatant after ultracentrifugation; S) and the insoluble fraction (pellet after ultracentrifugation; $P$ ) were analysed by Western blotting using anti-FlhF and anti-FlhG antibodies. (b) FlhF was immunoprecipitated with anti-FlhF antibody from the cytoplasmic fraction of the $\triangle f / h F G$ cells expressing both FlhF and FlhG (lane 2), followed by Western blotting. As a negative control, an immunoprecipitation using antiFlhF antibody with the $\triangle$ flhFG cells expressing only FlhG was also performed (lane 1). The band from the heavy chain of the anti-FlhF antibody was detected in lane 1 (indicated by an asterisk).

flagellar genes. In this study, larger amounts of the intermediate class proteins (FlgI and MotY) and the late class proteins (PomA, PomB, MotX and flagellins) were detected in the flhG mutant cells (Fig. 3). The results are consistent with the earlier reports of negative feedback regulation of intermediate-class gene expression by FlhG in V. cholerae (Correa et al., 2005) and by the FlhG homologue (FleN) in Pseudomonas (Dasgupta \& Ramphal, 2001). The lack of FlhG causes overexpression of the intermediate-class genes, including that of $\sigma^{28}$, leading to overexpression of the late-class genes. We speculate that multiple flagellation of the flh $G$ mutant cells requires the overexpression of flagellar genes.

FlhF, but not FlhG, seems to be involved in the polar placement of flagella. In electron microscopic observations, we were unable to find any flagellated $\Delta f l h F$ cells, but did find some $\Delta$ flhFG cells with multiple peritrichous flagella (Fig. 1c, d). Considering that the flhG-defective strain has multiple flagella at the pole (Fig. 1b), FlhF appears to be one of the determinants of the polar placement of the

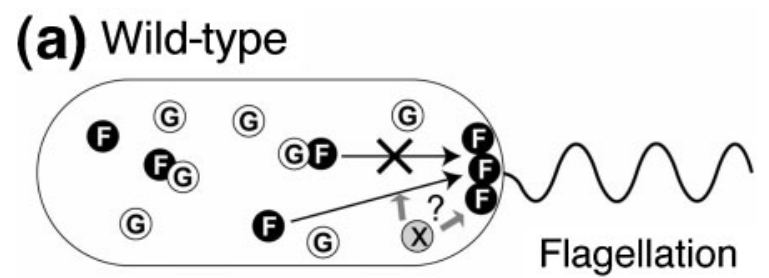

(b) FIhG overexpression

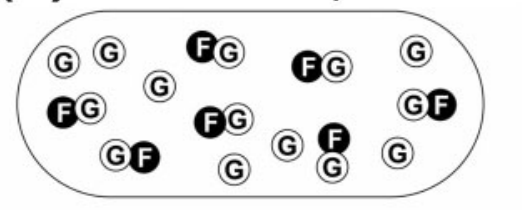

(c) FlhG depletion

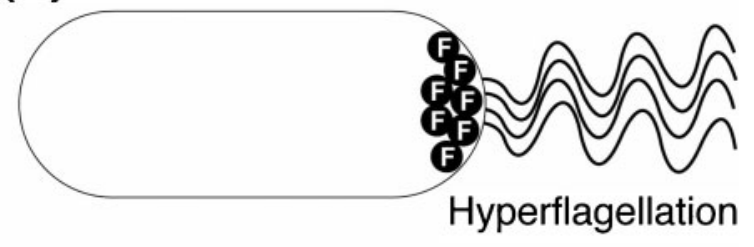

(E) FlhF (G) FIhG

Fig. 7. Working hypothesis for the regulation of the number of polar flagella by FlhF and FlhG in V. alginolyticus cells. (a) In a wildtype cell, FlhF is localized at the pole and promotes the assembly of the MS ring of flagella. FlhG in the cytoplasm inhibits FlhF localization at the pole by interacting with FlhF, and thus represses excessive flagellation. Therefore, the number of polar flagella is regulated to be one by FlhF and FlhG. FlhF may need a factor ' $X$ ' to localize at the pole, as discussed in the text. The factor may be involved in the polar localization of FlhF or in the stability of FlhF at the pole. (b) When FlhG is overexpressed, almost all of the FlhF molecules are captured by FlhG. FlhG-captured FlhF does not localize at the pole, and thus FlhG-overproducing cells fail to produce a polar flagellum. (c) When FlhG is depleted, most of the FlhF is localized at the pole, and thus the cells produce multiple flagella at the pole.

flagellum, which is consistent with results for $P$. aeruginosa and P. putida (Murray \& Kazmierczak, 2006; Pandza et al., 2000). If FlhF collaborates with FlhG, they could be colocalized in the cell, or each protein might affect the subcellular localization of the other. To verify the collaboration between FlhF and FlhG, we constructed GFP-fusion variants of both FlhF and FlhG, and observed their localization in the cell. FlhF-GFP was localized at the flagellated pole, while most of the FlhG was diffusely expressed throughout the cytoplasm (Fig. 4). Strikingly, FlhF-GFP localization was more intense in the $\Delta f l h F G$ cells, suggesting that FlhG may negatively affect the polar localization of FlhF (Fig. 5b). The effect of FlhG on the polar localization of FlhF was confirmed in a non- 
flagellated E. coli strain (data not shown). This leads to the hypothesis that FlhF and FlhG interact with each other to regulate flagellar number and placement. We were able to demonstrate an interaction between FlhF and FlhG by coimmunoprecipitation (Fig. 6). From these results, we propose a working hypothesis for the role of FlhF and FlhG in controlling polar flagellar number and placement (Fig. 7). FlhF works at the pole to determine the placement of the flagellum and promotes the expression of flagellar genes (Fig. 7a). FlhG interacts with FlhF and inhibits FlhF from localizing at the pole, resulting in the suppression of flagellation (Fig. 7a). Overproduction of FlhG reduces the polar localization of FlhF, such that FlhF diffuses throughout the cytoplasm, and results in a non-flagellated phenotype (Fig. 7b). Depletion of FlhG causes strong polar localization of FlhF, leading to hyperflagellation at the pole (Fig. 7c). Consequently, the number of polar flagella is controlled to allow the production of a single flagellum by the collaboration of FlhF and FlhG. FlhG seems to act not only as an anti-coactivator of $\sigma^{54}$, as reported in Pseudomonas, but also an inhibitor of FlhF localization.

FlhF has a GTP-binding motif and shows similarity to $E$. coli SRP receptor FtsY, which is a membrane-associated receptor that targets the SRP/ribosome-nascent-chain complex to the translocon. The dissociation of FtsY from SRP is regulated by GTP hydrolysis of FtsY and SRP (Shan \& Walter, 2005). We speculate that the FlhF-FlhG interaction is also regulated by GTP hydrolysis by analogy with FtsY. On the other hand, FlhG has an ATP-binding motif and shows similarity to E. coli MinD. The MinD dimer associates with the inner membrane and gathers MinC, which inhibits FtsZ polymerization, and thus inhibits the generation of the division plane (Rothfield et al., 2005; Shapiro et al., 2002). The MinD dimer dissociates into monomers by interacting with MinE, and monomeric MinD dissociates from the membrane, hydrolysing ATP. Therefore, it is possible that ATP hydrolysis mediates the interaction of FlhG with FlhF or with FlaK, which is the Vibrio homologue of Pseudomonas FleQ, and is similar to MinD.

Localization of FlhG-GFP was different in Vibrio and E. coli cells. FlhG-GFP diffused completely through the cytoplasm and was not localized at the pole in E. coli cells (data not shown), while FlhG-GFP was localized at the pole in some of the Vibrio cells transformed with the flhG$g f p$ plasmid (Fig. 5d, e). We also observed localization of FlhG-GFP in Vibrio rpoN $\left(\sigma^{54}\right)$ mutant cells (Fig. 5f). These results may indicate that FlhG is localized at the pole by a certain factor which is unique to Vibrio and whose gene is transcribed independently of $\sigma^{54}$. Polar localization of FlhF-GFP was observed in E. coli cells (data not shown) and in the rpoN mutant cells (Fig. 5c). It seems that FlhF by itself could recognize the pole and determine where the polar flagellum is generated. FlhF-GFP was not strongly localized at the pole in rpoN mutant cells (Fig. 5c), although rpoN mutant cells did not express $f h G$, which is expressed under the control of $\sigma^{54}$ (Fig. 3b). This implies that FlhF might require another factor to allow its localization at the cell pole. We know that FlhF is a key player in determining the localization of polar flagella; however, its mechanism is unclear. An investigation of the function of FlhF will lead to a better understanding of the mechanism of polar localization of flagella.

\section{ACKNOWLEDGEMENTS}

We thank Gillian Fraser for useful discussions and Sachi Tatematsu for assistance with the anti-FlhG antibody production. This work was supported in part by grants-in-aid for scientific research from the Hayashi Memorial Foundation for Female Natural Scientists (to A. K.), from the Ministry of Education, Science, and Culture of Japan, and from the Strategic International Cooperative Program of the Japan Science and Technology Agency (JST) (to M. H.).

\section{REFERENCES}

Brun, Y. V., Marczynski, G. \& Shapiro, L. (1994). The expression of asymmetry during Caulobacter cell differentiation. Annu Rev Biochem 63, 419-450.

Correa, N. E., Peng, F. \& Klose, K. E. (2005). Roles of the regulatory proteins FlhF and FlhG in the Vibrio cholerae flagellar transcription hierarchy. J Bacteriol 187, 6324-6332.

Dasgupta, N. \& Ramphal, R. (2001). Interaction of the antiactivator FleN with the transcriptional activator FleQ regulates flagellar number in Pseudomonas aeruginosa. J Bacteriol 183, 6636-6644.

Dasgupta, N., Arora, S. K. \& Ramphal, R. (2000). fleN, a gene that regulates flagellar number in Pseudomonas aeruginosa. J Bacteriol 182, $357-364$

Dasgupta, N., Ferrell, E. P., Kanack, K. J., West, S. E. \& Ramphal, R. (2002). fleQ, the gene encoding the major flagellar regulator of Pseudomonas aeruginosa, is $\sigma^{70}$ dependent and is downregulated by Vfr, a homolog of Escherichia coli cyclic AMP receptor protein. J Bacteriol 184, 5240-5250.

Dasgupta, N., Wolfgang, M. C., Goodman, A. L., Arora, S. K., Jyot, J., Lory, S. \& Ramphal, R. (2003). A four-tiered transcriptional regulatory circuit controls flagellar biogenesis in Pseudomonas aeruginosa. Mol Microbiol 50, 809-824.

Fukuoka, H., Yakushi, T., Kusumoto, A. \& Homma, M. (2005). Assembly of motor proteins, PomA and PomB, in the $\mathrm{Na}^{+}$-driven stator of the flagellar motor. J Mol Biol 351, 707-717.

Grant, S. G., Jessee, J., Bloom, F. R. \& Hanahan, D. (1990). Differential plasmid rescue from transgenic mouse DNAs into Escherichia coli methylation-restriction mutants. Proc Natl Acad Sci U S A 87, 4645-4649.

Guzman, L. M., Belin, D., Carson, M. J. \& Beckwith, J. (1995). Tight regulation, modulation, and high-level expression by vectors containing the arabinose PBAD promoter. J Bacteriol 177, 4121-4130.

Harwood, C. S., Fosnaugh, K. \& Dispensa, M. (1989). Flagellation of Pseudomonas putida and analysis of its motile behavior. J Bacteriol 171, 4063-4066.

Kawagishi, I., Okunishi, I., Homma, M. \& Imae, Y. (1994). Removal of the periplasmic DNase before electroporation enhances efficiency of transformation in a marine bacterium Vibrio alginolyticus. Microbiology 140, 2355-2361.

Kawagishi, I., Nakada, M., Nishioka, N. \& Homma, M. (1997). Cloning of a Vibrio alginolyticus rpoN gene that is required for polar flagellar formation. J Bacteriol 179, 6851-6854. 
Kearns, D. B. \& Losick, R. (2003). Swarming motility in undomesticated Bacillus subtilis. Mol Microbiol 49, 581-590.

Kubori, T., Shimamoto, N., Yamaguchi, S., Namba, K. \& Aizawa, S. (1992). Morphological pathway of flagellar assembly in Salmonella typhimurium. J Mol Biol 226, 433-446.

Kubori, T., Yamaguchi, S. \& Aizawa, S. (1997). Assembly of the switch complex onto the MS ring complex of Salmonella typhimurium does not require any other flagellar proteins. I Bacteriol 179, 813-817.

Kusumoto, A., Kamisaka, K., Yakushi, T., Terashima, H., Shinohara, A. \& Homma, M. (2006). Regulation of polar flagellar number by the $f l h F$ and flh $G$ genes in Vibrio alginolyticus. J Biochem 139, 113-121.

Macnab, R. (1996). Flagella and motility, In Escherichia coli and Salmonella, pp. 123-145. Edited by F. C. Neidhardt. Washington, DC: American Society for Microbiology.

Macnab, R. M. (2004). Type III flagellar protein export and flagellar assembly. Biochim Biophys Acta 1694, 207-217.

McCarter, L. L. (2001). Polar flagellar motility of the Vibrionaceae. Microbiol Mol Biol Rev 65, 445-462.

Millikan, D. S. \& Ruby, E. G. (2004). Vibrio fischeri flagellin A is essential for normal motility and for symbiotic competence during initial squid light organ colonization. J Bacteriol 186, 4315-4325.

Murray, T. S. \& Kazmierczak, B. I. (2006). FlhF is required for swimming and swarming in Pseudomonas aeruginosa. J Bacteriol 188, 6995-7004.

Nambu, T. \& Kutsukake, K. (2000). The Salmonella FlgA protein, a putative periplasmic chaperone essential for flagellar $\mathrm{P}$ ring formation. Microbiology 146, 1171-1178.

Niehus, E., Gressmann, H., Ye, F., Schlapbach, R., Dehio, M., Dehio, C., Stack, A., Meyer, T. F., Suerbaum, S. \& Josenhans, C. (2004). Genomewide analysis of transcriptional hierarchy and feedback regulation in the flagellar system of Helicobacter pylori. Mol Microbiol 52, 947-961.

Nishioka, N., Furuno, M., Kawagishi, I. \& Homma, M. (1998). Flagellin-containing membrane vesicles excreted from Vibrio alginolyticus mutants lacking a polar-flagellar filament. J Biochem 123, $1169-1173$.
Okunishi, I., Kawagishi, I. \& Homma, M. (1996). Cloning and characterization of $\operatorname{mot} Y$, a gene coding for a component of the sodium-driven flagellar motor in Vibrio alginolyticus. J Bacteriol 178, 2409-2415.

Pandza, S., Baetens, M., Park, C. H., Au, T., Keyhan, M. \& Matin, A. (2000). The G-protein FlhF has a role in polar flagellar placement and general stress response induction in Pseudomonas putida. Mol Microbiol 36, 414-423.

Prouty, M. G., Correa, N. E. \& Klose, K. E. (2001). The novel $\sigma^{54}$ - and $\sigma^{28}$-dependent flagellar gene transcription hierarchy of Vibrio cholerae. Mol Microbiol 39, 1595-1609.

Rothfield, L., Taghbalout, A. \& Shih, Y. L. (2005). Spatial control of bacterial division-site placement. Nat Rev Microbiol 3, 959-968.

Shan, S. O. \& Walter, P. (2005). Co-translational protein targeting by the signal recognition particle. FEBS Lett 579, 921-926.

Shapiro, L., McAdams, H. H. \& Losick, R. (2002). Generating and exploiting polarity in bacteria. Science 298, 1942-1946.

Studier, F. W. (1991). Use of bacteriophage T7 lysozyme to improve an inducible T7 expression system. J Mol Biol 219, 37-44.

Terashima, H., Fukuoka, H., Yakushi, T., Kojima, S. \& Homma, M. (2006). The Vibrio motor proteins, MotX and MotY, are associated with the basal body of $\mathrm{Na}^{+}$-driven flagella and required for stator formation. Mol Microbiol 62, 1170-1180.

Tsuda, M. \& lino, T. (1983). Transductional analysis of the flagellar genes in Pseudomonas aeruginosa. J Bacteriol 153, 1018-1026.

Wu, J. \& Newton, A. (1997). Regulation of the Caulobacter flagellar gene hierarchy; not just for motility. Mol Microbiol 24, 233-239.

Xu, M., Yamamoto, K., Honda, T. \& Ming, X. (1994). Construction and characterization of an isogenic mutant of Vibrio parahaemolyticus having a deletion in the thermostable direct hemolysin-related hemolysin gene (trh). J Bacteriol 176, 4757-4760.

Yagasaki, J., Okabe, M., Kurebayashi, R., Yakushi, T. \& Homma, M. (2006). Roles of the intramolecular disulfide bridge in MotX and MotY, the specific proteins for sodium-driven motors in Vibrio spp. $J$ Bacteriol 188, 5308-5314.

Edited by: P. W. O'Toole 